\title{
DESCRIPTION DE DEUX ESPÈCES NOUVELLES
}

\section{DU GENRE TRIATOMA : T. CARRIONI N. SP.,}

\section{ET T. PINTOI N. SP.}

\author{
Par F. LARROUSSE
}

M. le professeur Brumpt a bien voulu me confier l'étude de deux espèces du genre Triatoma, qui lui ont été offertes très aimablement par M. le professeur Howard, directeur du National Museum de Washington. Les différentes diagnoses des espèces déjà connues ne pouvant s'appliquer aux exemplaires qui font l'objet de la présente note, je les décrirai sous les noms spécifiques nouveaux de Triatoma carrioni et de $T$. pintoi.

\section{Triatoma carrioni n. sp.}

TAILlE. - Espèce de moyenne grandeur (fig. 1) :

Longueur : 2 cm., 1 à $2 \mathrm{~cm} ., 2\left\{\begin{array}{llll}\text { Tête } \ldots \ldots \ldots \ldots & 0 & \mathrm{~cm} ., 4 \\ \text { Pronotum } & \ldots \ldots \ldots & 0 & \mathrm{~cm} ., 32 \\ \text { Scutellum } \ldots \ldots \ldots & 0 & \mathrm{~cm} ., 20 \\ \text { Abdomen } & \ldots \ldots \ldots & 1 & \mathrm{~cm} ., 15\end{array}\right.$

Largeur : 0 cm., 8.

Coloration générale. - Fond sépia tirant sur le noir ; taches orangées sur le thorax, la pointe du scutellum, les cories et le connexivum.

TÊTE. - Grêle, allongée, finement granuleuse, à pilosité très courte ; partie anté-oculaire trois fois plus longue que la postoculaire. Lobe médian fortement caréné, légèrement plus long que les lobes latéraux ; en arrière du lobe médian, crête saillante sur le vertex de la tête jusqu'au niveau des ocelles, crête délimitée de chaque côté d'une façon très nette par une fine ligne noire non granuleuse. Antennes longues; elles s'insèrent sur un tubercule court situé à égale distance de l'apex de la tête et de l'œil ; premier article court, n'atteignant pas l'extrémité antérieure des lobes latéraux, deuxième article trois fois plus long que le premier, troisième article légèrement plus court que le précédent, le quatrième article manque; les trois articles présents sont de coloration noire.

Annales de Parasitologie, T. IV, $\mathrm{N}^{\circ} 2 .-1^{\mathrm{er}}$ avril 1926, p. 136-139. 
Yeux saillants. Rostre long, recouvrant largement le bord antérieur du thorax, $1^{\text {er }}$ et $3^{\circ}$ articles sensiblement de même longueur. le $1^{\text {er }}$ vu de profil n'atteint pas l'extrémité apicale du tubercule antennifère, le $2^{\circ}$ article est trois fois plus long que le $3^{\circ}$.

Thorax. - Pronotum un peu plus court que la tête. Lobe antérieur réduit, divisé à sa partie médiane par une suture plus prononcée en arrière ; l'ensemble de ce lobe est constitué par des

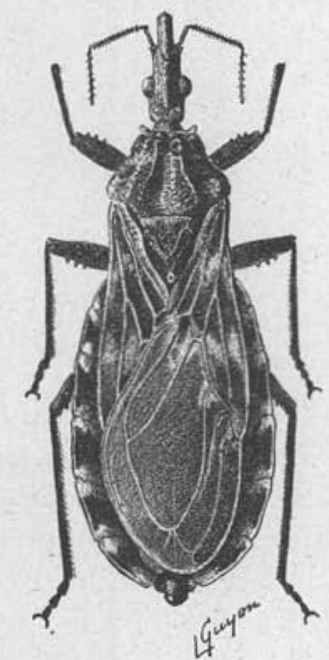

Fig. 1. - Triatoma carrioni, Q n. sp., $\times 3$.

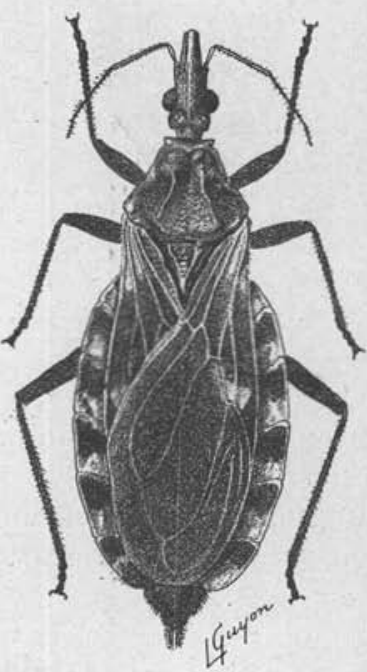

Fig. 2. - Triatoma pintoi, Q n. sp.. $\times 3,3$.

tubercules plats de coloration noirâtre ; le bord antérieur convexe est limité de chaque côté par un tubercule saillant de couleur orangée. Le lobe postérieur élargi, trois fois plus long que le lobe antérieur, présente de chaque côté de la ligne médiane deux carènes très atténuées en arrière. Le fond du lobe postérieur est chagriné, de coloration orangée, avec quatre taches noires, deux sur les côtés qui n'atteignent pas la bordure, les deux autres plus volumineuses embrassent les trois-quart postérieurs des deux carènes en les débordant largement. Scutellum triangulaire avec deux carènes qui délimitent un deuxième triangle; pointe mousse, excavée, en forme de cuillère, de couleur orangée (fig. 3 ).

Aвdomen. - Noir sépia. Hémélytres atteignant l'extrémité de l'abdomen, chez le $\delta$, non chez la $q$; cories noires, avec deux taches orangées sinueuses et étroites ; membranes enfumées, à nervures 
plus claires, orangées au voisinage des cories. Le connexivum déborde modérément les hémélytres, le bord basal et apical des segments est de couleur orangée.

Pattes. - Noires; fémurs armés de trois épines très nettes et espacées sur leur bord inférieur (fig. 4).

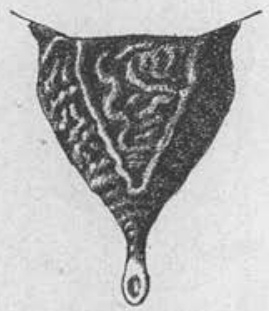

Fig. 3. - Triatoma carrioni, scutellum.

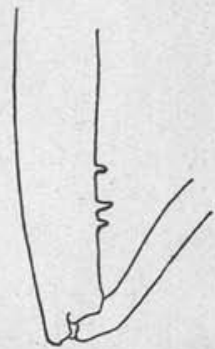

Fig. 4. - Triatoma carrioni, fémur antérieur droit.

Haвitat. - Loja, Equateur, $2 \delta, 2 q$; exemplaires communiqués par le professeur Campos, de Guayaquil, au National Museum de Washington. Je suis heureux de dédier cette espèce à $M$. Clodoneo Carrion, le distingué naturaliste qui a capturé ces exemplaires à Loja, en 1924.

Triatoma pintoi, n. sp.

TAILlE. - Espèce de petite taille (fig. 2) :

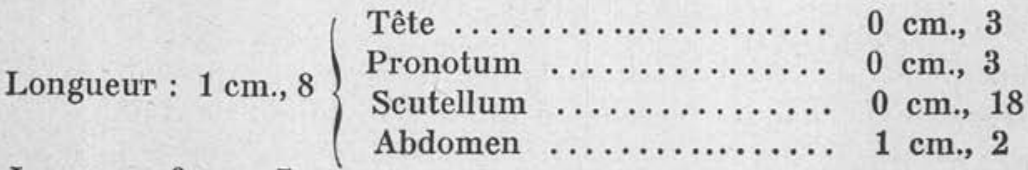

Largeur : $0 \mathrm{~cm} ., 7$.

Coloration GÉnÉRALE. - Brun-noir ; taches rouge-ocre sur le thorax, les hémélytres et le connexivum.

TÊTE: - Courte et grêle, granuleuse, la partie anté-oculaire deux fois et demi plus longue que la post-oculaire. Lobe médian modérément caréné, légèrement plus long que les lobes latéraux. Antennes courtes : elles s'insèrent sur un tubercule court, situé plus près des yeux que de l'apex de la tête ; $1^{\text {er }}$ article court, n'atteignant pas l'extrémité antérieure des lobes latéraux, $2^{\circ}$ article trois fois plus long que le $1^{\text {er }}, 3^{\circ}$ article de la longueur du $2^{\circ}, 4^{e}$ article légèrement plus court que les deux précédents. Yeux peu saillants. 
Rostre long, de coloration plus claire que le reste de la tête, le $1^{\text {er }}$ article vu de profil atteint le niveau de l'extrémité apicale du tubercule antennifère, le $2^{\circ}$ article dépasse la longueur de la tête, le $3^{\circ}$ très court, se loge entre les deux hanches de la $1^{\text {re }}$ paire de pattes.

Thorax. - Pronotum de longueur sensiblement égale à celle de la tête. Lobe antérieur peu distinct, sans relief très caractérisé. Le lobe postérieur peu évasé, présente de chaque côté de la ligne médiane deux carènes étroites. Le bord antérieur, les tubercules antéro-externes, les marges latérales et les angles postéro-externes sont limités par une étroite bordure rouge-ocre. Scutellum noir, à pointe mousse et non relevée.

Aвdomen. - Brun-noir en-dessous, sans trace de pilosité. Cories brunes, avec une tache rougeâtre à l'apex, deuxième tache moins nette sur le bord inférieur près du milieu; membranes brunes à nervures peu visibles. Connexivum assez large, chaque segment présente une tache rouge-ocre sur son bord apical et basal, cette dernière est beaucoup plus large.

Pattes. - Noires, sans caractères très tranchés.

Haвiтat. - Lauderdale, Floride, $2 \delta, 2 q$; exemplaires récoltés par M. R.-H. Bloom. Je dédie cette espèce à mon collègue Cesar Pinto, auteur de plusieurs travaux, intéressant la systématique du genre Triatoma.

Cette espèce est très voisine de Triatoma sanguisuga (Lecomte) ; elle se distingue de cette dernière espèce par sa taille, beaucoup plus réduite, son aspect général plus grêle, son thorax moins dilaté en arrière.

\section{RÉSUMÉ}

Je décris dans cette note, deux espèces nouvelles du genre Triatoma: Triatoma carrioni, provenant de la république de l'Equateur et Triatoma pintoi, espèce provenant de la Floride.

Laboratoire de Parasilologie de la Faculté de médecine de Paris. 